\title{
Plasmonic Effects in Tin Disulfide Nanostructured Thin Films Obtained by the Close-Spaced Vacuum Sublimation
}

\author{
M.O. Stetsenko ${ }^{1}$ - A.A. Voznyi $^{2}$ • V.V. Kosyak ${ }^{2}$ - S.P. Rudenko ${ }^{1}$ L.S. Maksimenko ${ }^{1}$. \\ B.K. Serdega ${ }^{1}$ - A.S. Opanasuk ${ }^{2}$
}

Received: 11 July 2016 / Accepted: 29 August 2016

(C) Springer Science+Business Media New York 2016

\begin{abstract}
This work reports the investigation of plasmonic effects in tin disulfide $\left(\mathrm{SnS}_{2}\right)$ nanostructured films obtained by the close-spaced vacuum sublimation method (CSS). Structural properties and phase composition of $\mathrm{SnS}_{2}$ films were studied with the help of field emission scanning electron microscopy (FE-SEM), atomic force microscopy (AFM), Xray diffraction (XRD) and Raman spectroscopy. Surface morphology and optical polarization properties of $\mathrm{SnS}_{2}$ films deposited at different substrate temperature were investigated by modulation-polarization spectroscopy (MPS). Surface plasmon resonances (SPR) with localized and polariton types were observed by measuring of angular and spectral characteristics of polarization difference. Radiative and nonradiative modes of surface plasmons have been analyzed at different light incident angles. The influence of surface morphology on resonant parameters of different types of SPR was studied. Correlation between the experimental results and theoretical calculations was established. The refractive and absorption indexes were found for the $\mathrm{SnS}_{2}$ films at different substrate temperature.
\end{abstract}

Keywords Tin disulfide $\cdot$ Nanostructured films · Surface plasmon resonance $\cdot$ Modulation-polarization spectroscopy

M.O. Stetsenko

stetsenkomax@gmail.com

S.P. Rudenko

rudenko.svetlana@gmail.com

1 V. Lashkaryov Institute of Semiconductor Physics, NAS of Ukraine, 45, Nauky Pr, Kyiv 03028, Ukraine

2 Sumy State University, 2, Rymsky Korsakov Str, Sumy 40007, Ukraine

\section{Introduction}

Tin disulfide thin film is of a great interest as a promising material for applications in optoelectronics. In particular, due to high mobility of free carriers [1], n-type of conductivity and band gap of $2.2 \mathrm{eV}$ [2] this compound could be considered as an alternative to conventional $\mathrm{CdS}$ solar cells window material [3]. Also, the $\mathrm{SnS}_{2}$ is a layered 2-D semiconductor, which makes it possible to use it in a high-speed photo-detectors and as a catalyst in Li-ion batteries [4-6].

However, it is known that $\mathrm{SnS}_{2}$ films have strong anisotropy of optical properties, which complicates its application in electronics devices [6]. One of the possibilities to improve the efficiency of solar cells is an enhancement of light absorption due to surface plasmon resonance (SPR) [7]. For example, as was shown in [8], the deposition of plasmonic metal nanoparticles (grains, clusters) on a base layer leads to increasing of absorption range of solar radiation. The essence of SPR phenomenon is based on plasmon absorption in the visible, near UV and IR wavelength range. Experimental detection of SPR is related to measuring of optical transmission and extinction. In this case the resonant excitations of surface plasmon-polariton (SPP) (on an infinite flat metal-dielectric surface) or localized surface plasmon (LSP) (on separated non-interacting grains and between metal grains due to electrodynamic/dipole-dipole interactions) takes place. For nanostructured materials, the SPR appears due to their optical conductivity or due to the presence of metal nanoparticles like gold nanoparticles embedded in a dielectric/ semiconducting medium like tin dioxide $\left(\mathrm{SnO}_{2}\right)$ and tin disulfide $\left(\mathrm{SnS}_{2}\right)$ [7, 8]. In our work [9], the SPR was detected for nanostructured $\mathrm{SnO}_{2}$ films without containing metal nanoparticles.

Plasmonic semiconductors are modern perspective nanomaterials that scientists intensively study nowadays 
[10-12]. Doped semiconductors $\mathrm{Si}, \mathrm{SeGe}, \mathrm{GaAs}, \mathrm{GaN}, \mathrm{ZnO}$ and indium tin oxide (ITO) can be alternative plasmonic materials [10]. Size-dimension reduction of plasmonic semiconductors to nanocrystals (NCs) leads to the tunable optical response from visible to infrared range, because their free carrier density can be tuned over several orders of magnitude. For copper chalcogenide NCs, also called the "self-doped semiconductors", the oxidation tuning of the near-infrared (NIR) plasmonic absorption can be achieved by the ambient oxygen under different temperatures or metal salt solutions $[11,13]$.

Manifestation of the plasmon resonances in twodimensional (2D) semiconductor compounds (2D metal oxides and dichalcogenides) is desirable for many applications. Reduction of the material thickness in 2D materials can however lead to alterations in their plasmon dispersion relation $[14,15]$. Plasmon dispersion is highly dependent on the geometrical configuration of the nanostructures $[16,17]$. The existence of one large depolarization factor in the thickness axis of 2D materials can be used to tune the plasmon resonance response into the near UV, visible and nearinfrared (NIR) regions for structures lithium into 2D molybdenum disulfide $\left(\mathrm{MoS}_{2}\right)$ nanoflakes demonstrated in [14, 18]. In the experimental work [19] two plasmon resonance peaks associated with the thickness and the lateral dimension axes were observed in the exfoliating 2D molybdenum oxide $\left(\mathrm{MoO}_{3}\right)$. [19]. Plasmonic positions of these peaks could be tuned by the choice of the solvent. Hybrid nanostructures of metal/two-dimensional nanomaterials reviewed in detail in [20].

That is why one can expect similar plasmonic effects in nanostructured $\mathrm{SnS}_{2}$ films. The knowledge about optical properties for $\mathrm{SnS}_{2}$ films of nanosized thickness is limited. The UV-Vis-NIR transmission and reflectance spectra of the ultrathin $\mathrm{SnS}_{\mathrm{x}}$ films on quartz substrates with thickness less than $100 \mathrm{~nm}$ were investigated in works [21,22].

The aim of this work is to study the plasmonic effects in the nanostructured $\mathrm{SnS}_{2}$ thin films deposited by CSS method utilizing the modulation-polarization spectroscopy (MPS) technique. This optical technique is effective for diagnostics and characterization of the SPR features in nanosized films of noble metals and metal-dielectric nanocomposites [9, 23-26]. The dependence of plasmonic effects on surface morphology and structural features is studied by measuring of parameter of the polarization difference $\rho(\theta, \lambda, d)$, where $\theta, \lambda, d$ - the incident angle of light, wavelength and sample thickness, respectively. The modification of surface morphology of $\mathrm{SnS}_{2}$ films was achieved by varying the substrate temperature. For investigation of the structural and optical properties we used a combination of different analytical techniques such as: field emission scanning electron microscopy (FE-SEM), X-ray analysis, Raman spectroscopy and MPS.

\section{Experimental Details}

\section{Samples Preparation}

The $\mathrm{SnS}_{2}$ films were obtained by the CSS method in a vacuum chamber analogously to our previous work [27]. The detailed description of the method for thin films growth is given in [28]. The stoichiometric $\mathrm{SnS}_{2}$ powder was used for evaporation. The thin films were deposited on ultrasonically cleaned glass substrate. The evaporator temperature was constant at $T_{e}=675^{\circ} \mathrm{C}$, while substrate temperature $T_{s}$ was varied from 175 to $275^{\circ} \mathrm{C}$. The deposition time was $4 \mathrm{~min}$.

\section{Surface Morphology and Structural Studies}

Surface morphology of the films was investigated using an FEI Nova NanoSEM 650 Schottky field emission scanning electron microscope (FE-SEM). The chemical composition analysis of the $\mathrm{SnS}_{2}$ samples was carried out by Apollo Xenergy dispersive spectroscope (EDS) using standardless energy techniques. The parameters of EDS experiment were as follows: an accelerating voltage of $15 \mathrm{kV}$, a detector resolution of $125.4 \mathrm{eV}$, a working distance of $7 \mathrm{~mm}$, and a spot size of 5.5. The concentrations of elements were determined by averaging of results for at least 10 measurements from different points on the surface.

In order to study thickness of the $\mathrm{SnS}_{2}$ films the NT-MDT atomic force microscope (AFM) in a semi-contact mode was used.

The study of the structural properties and phase composition of the films was carried out with the help of a DRON-4-07 diffractometer. $\mathrm{CuK}_{\alpha}$ radiation and Bragg-Brentano configuration were used. The range of the $2 \theta$ angle was varied from $10^{\circ}$ to $80^{\circ}$. Identification of the crystal phases was performed with the Crystallography Open Database (COD).

Raman spectra were measured by using Renishaw's InVia 90 V727 Raman spectrometer at room temperature. An Ar ion laser with a wavelength of $514 \mathrm{~nm}$ was used as the excitation source. The diameter of the laser spot was $0.7 \mu \mathrm{m}$, the power density of the laser beam was $6.78 \mathrm{~W} / \mathrm{cm}^{2}$, and the accumulation time was $80 \mathrm{~s}$.

\section{Optical Polarization Studies}

The measurements of angular and spectral characteristics of polarization difference $\rho(\theta, \lambda)$ in Kretschmann geometry were performed by the modulation-polarization spectroscopy technique. The detailed description of the experimental setup is presented in [23]. The MPS technique is based on the modulation of polarization of electromagnetic radiation when the parallel $(p)$ and perpendicular $(s)$ polarizations relative to the incident plane are alternately transformed at a constant intensity, frequency, phase, and wave vector. A diffraction 
monochromator MDR-4 served as a source of spectral radiation in the wavelength range $(\lambda)$ from 0.4 to $1 \mu \mathrm{m}$. A photoelastic polarization modulator (PEPM) acted as a dynamic phase plate. Alternating phase incursion was caused by compression/expansion of the quartz plate. A quarterwave mode was selected by a proper supplying voltage. As a result, linear polarization was transformed into alternating right-to-left circular polarization. A stationary quarter-wave phase plate (PP) was placed after the PEPM. By rotating PP around the optical axis, a position of $\mathrm{PP}$ was selected at which polarization azimuths of radiation after PP alternated between $s$ - and $p$-polarization. The output radiation was directed at a photodetector PD (silicon photodiode). Reflected light in our case from the system of quartz half-cylinder-SnS $\mathrm{S}_{2}$ film-air was a measure of the difference of orthogonally polarized intensities, which was transformed by PD into alternating signal. This signal was registered by a selective amplifier equipped with a phase-lock detector (lock-in-voltmeter) tuned to the modulation frequency of $f=60 \mathrm{kHz}$. The variable component value is a magnitude of the difference of internal reflection coefficients of $s$ - and $p$-polarizations $\rho=\left(R_{s}{ }^{2}\right.$ $\left.R_{p}{ }^{2}\right) \sin (\omega t)$. The resulting value is the polarization difference $\rho(\lambda, \theta)=R_{s}{ }^{2}-R_{p}{ }^{2}$. According to the conventional terminology in polarimetry, the parameter $\rho$ is a $Q$-component of the Stokes vector.

\section{Results and Discussion}

\section{Morphology and Structural Studies}

Figure 1 shows FE-SEM images of $\mathrm{SnS}_{2}$ films obtained at different substrate temperatures $T_{s}$. It was found that thin films deposited at $175^{\circ} \mathrm{C}$ contain irregular grains with the average size of $100 \mathrm{~nm}$. With increasing of substrate temperature up to $250{ }^{\circ} \mathrm{C}$ the platelet grains of size about $150 \mathrm{~nm}$ are formed on the surface of the thin film. The distribution density of such grains considerably increases with further increasing of substrate temperature to $275^{\circ} \mathrm{C}$. Thus, it was established that crystallinity of the films increases with increasing of substrate temperature. The chemical composition of thin films was found to be $\delta=0.56$, where $\delta$ is the ratio of $\mathrm{Sn}$ to $\mathrm{S}$ concentration. This corresponds to the $\mathrm{Sn}$-rich $\mathrm{SnS}_{2}$ since the stoichiometric composition of $\mathrm{SnS}_{2}$ was reported to be $\delta=0.49[29]$.

The thickness of the samples measured with the help of AFM was found to be $50 \mathrm{~nm}$.

The XRD patterns of the thin films are presented in Fig. 2. As could be seen, the XRD patterns show only one peak at about $14.82 \theta$ degrees. This peak corresponds to the $(001)$ plane of hexagonal 2H-SnS 2 polytype [COD 96-900-0614]. The full width at half maximum (FHWM) of this peak for the
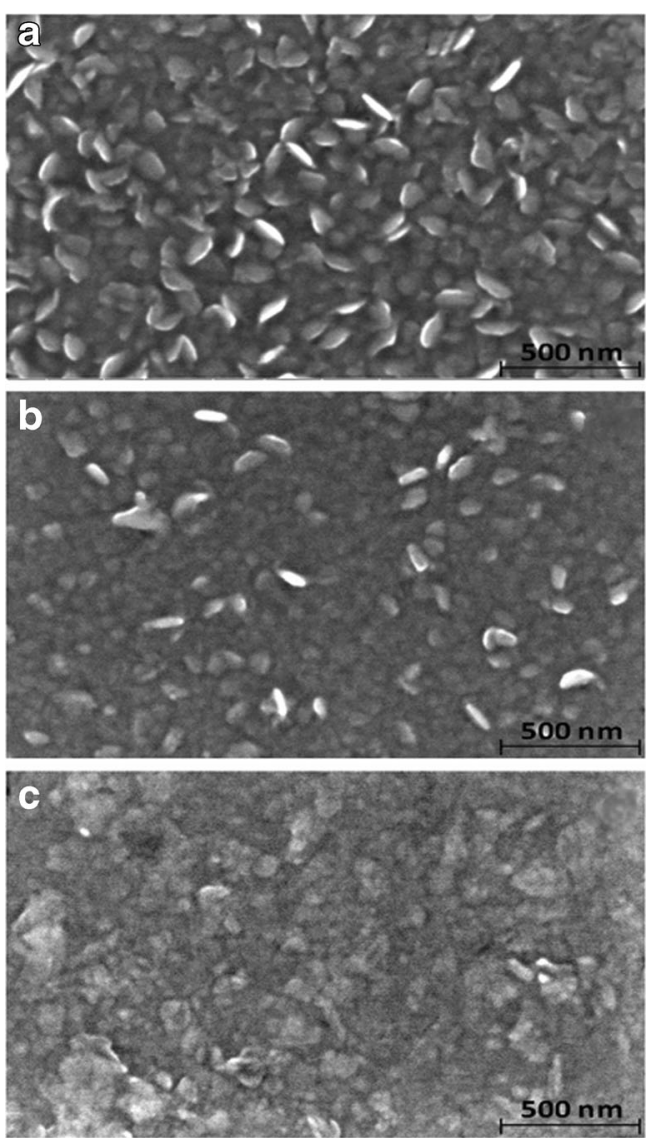

Fig. 1 FE-SEM images of $\mathrm{SnS}_{2}$ films surface with different substrate temperatures $T_{s}$ : a $275^{\circ} \mathrm{C}, \mathbf{b} 250^{\circ} \mathrm{C}$ and $\mathbf{c} 175^{\circ} \mathrm{C}$

sample obtained at $275^{\circ} \mathrm{C}$ are decreased and relative intensity was significantly higher than for other samples.

In additional, the results of coherent scattering domain (CSD) size by using the Scherrer equation were obtained. It has been observed that the largest CSD size of $16 \mathrm{~nm}$ has film obtained at $275^{\circ} \mathrm{C}$. The samples deposited at lower substrate temperatures of 175 and $250{ }^{\circ} \mathrm{C}$ have slightly less value of 15 and $11 \mathrm{~nm}$, respectively. In comparison with our previous work [27] for $1 \mu \mathrm{m}$ thickness $\mathrm{SnS}_{2}$ films the results of CSD for the directions perpendicular to the (001) crystallographic plane in some cases values were almost two times higher. This could be associated with the presence of different types of extended defects (subgrains, dislocation and stacking faults) in the crystal structure and phase features of the films.

Obtained results indicates improved crystal quality of the sample deposited at $275^{\circ} \mathrm{C}$. It should be noted that this finding is consistent with the results obtained by FE-SEM method.

The Raman spectroscopy is a one of the most effective method for the phase analysis of tin chalcogenides [30, 31]. This method usually used along with XRD and allows reliable identification of secondary phases and polytypes [32, 33]. In a case of single crystals the frequencies of the $\mathrm{SnS}_{2}, \mathrm{Sn}_{2} \mathrm{~S}_{3}$ and SnS- related Raman modes are well defined [30, 34-36]. However, Raman analysis of polycrystalline thin films could 


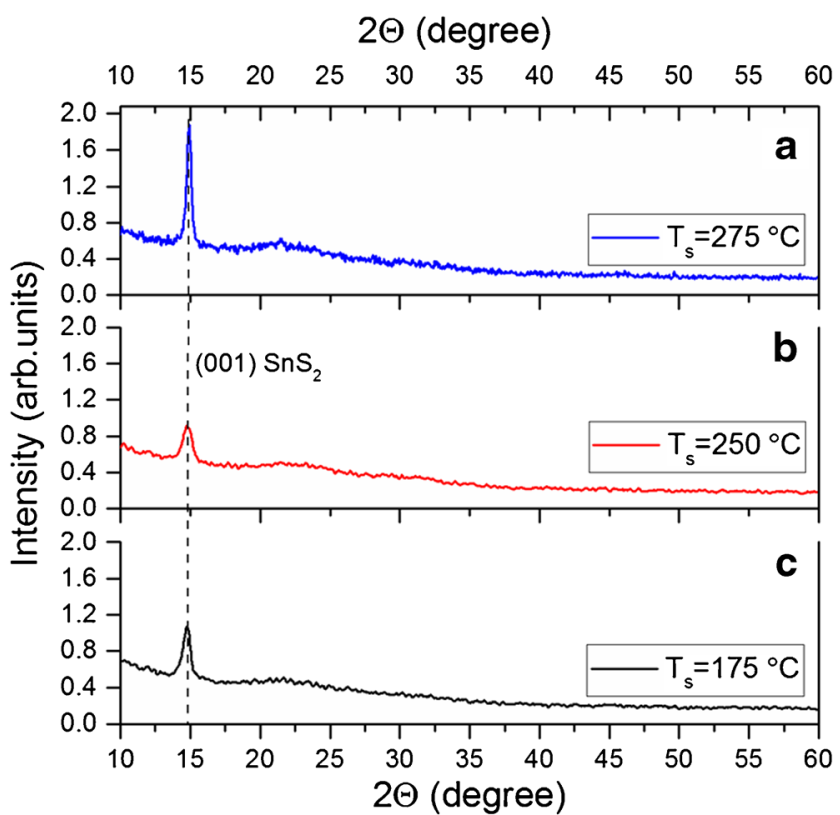

Fig. 2 XRD structural analysis for $\mathrm{SnS}_{2}$ films with different substrate temperature $T_{s}=275,250,175{ }^{\circ} \mathrm{C}$ (Lines are guides for the eyes)

be more complicated with broadening and shift of the Raman peaks due to grain boundaries, extended defects and stresses $[37,38]$. The Raman spectra of the thin films are presented on Fig. 3. The dominant peak at 314.6 corresponds to the $\mathrm{A}_{1 \mathrm{~g}}$ mode of $2 \mathrm{H}-\mathrm{SnS}_{2}$ [36]. For the samples obtained at a substrate temperature of 175 and $275{ }^{\circ} \mathrm{C}$ the low-intensity modes at 225,183 , and $93 \mathrm{~cm}^{-1}$ as well as mode at $154 \mathrm{~cm}^{-1}$ which could be assigned to $A_{1 \mathrm{~g}}$ and $\mathrm{B}_{2 \mathrm{~g}}$ symmetry of the $\mathrm{SnS}$ phase, respectively, was observed [30]. Also, the weak mode at $234 \mathrm{~cm}^{-1}$ corresponds to the $\mathrm{A}_{1 \mathrm{~g}}$ symmetry of the $\mathrm{Sn}_{2} \mathrm{~S}_{3}$ phase [34]. As could be seen from Fig. 3b, the relative intensity of $\mathrm{SnS}$ and $\mathrm{Sn}_{2} \mathrm{~S}_{3}$-related modes on the Raman spectrum of the sample obtained at $250{ }^{\circ} \mathrm{C}$ is increased. In particular, the intensive modes of the $\mathrm{A}_{1 \mathrm{~g}}$ symmetry at 93 and $183 \mathrm{~cm}^{-1}$, as well as mode, which could be assigned with the $\mathrm{B}_{2 \mathrm{~g}}$ symmetry of $\mathrm{SnS}$ phase or $\mathrm{A}_{1 \mathrm{~g}}$ symmetry of $\mathrm{Sn}_{2} \mathrm{~S}_{3}$ phase, were detected. Additionally, the SnS-related weak peak of $\mathrm{B}_{2 \mathrm{~g}}$ symmetry at $290 \mathrm{~cm}^{-1}$ was detected [30]. This indicates the highest amount of the $\mathrm{SnS}$ and $\mathrm{Sn}_{2} \mathrm{~S}_{3}$ secondary phases in a sample obtained at $250^{\circ} \mathrm{C}$ among all samples. Additionally, it could be associated with phase transition from $\mathrm{SnS}_{2}$ to $\mathrm{Sn}_{2} \mathrm{~S}_{3}$ and $\mathrm{SnS}$ phase due to evaporation of sulfur.

\section{Optical Polarization Studies}

For $\mathrm{SnS}_{2}$ films at $T_{s}=175^{\circ} \mathrm{C}$ Fig. 4 shows the spectral characteristics of polarization difference $\rho(\lambda)$ in a different range of incident angles $\theta$ relative to the critical angle of the total internal reflection, i.e. $\theta_{c r}=43.6^{\circ}$. In Fig. 4 a the bands of $\rho(\lambda)$ have a broad complex contour. For each curve of $\rho(\lambda)$ two extrema are observed, which are blue-shifted relative to each

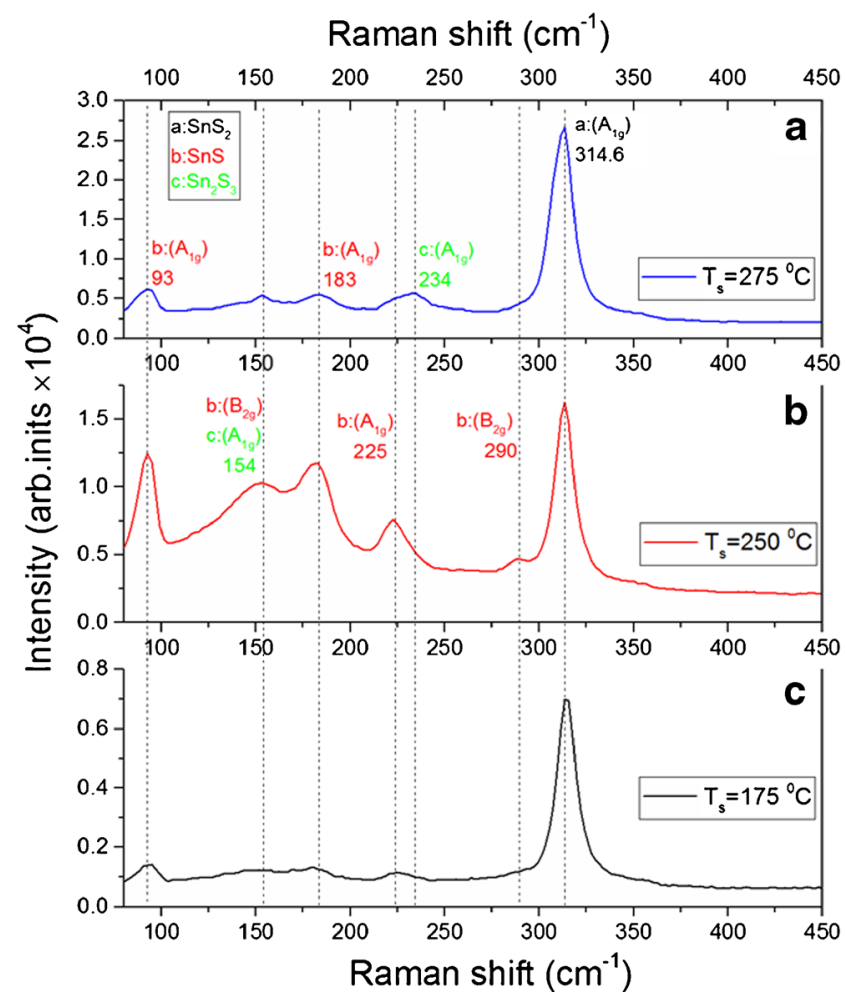

Fig. 3 Raman spectra of $\mathrm{SnS}_{2}$ films with different substrate temperature $T_{S}=275,250,175^{\circ} \mathrm{C}$. (Lines are guides for the eyes)

other with increasing angle $\theta$. Its origin is determined by surface plasmon's excitations. The SPR occurs, when the phase synchronism condition is satisfied and both frequency $(\omega)$ and wave vector $(k)$ of light excitation match those of the surface plasmon's frequency and wave vector. An opposite sign of amplitude of $\rho(\lambda)$ at phase-locked detection is caused by different type of SPR as a result of different interaction of $s$ - and $p$-polarized light with conduction electrons of the films. In particular, the extremum at negative value of $\rho(\lambda)$ in a shortwavelength range around $\lambda_{L S P} \sim 500 \mathrm{~nm}$ indicates to the resonant excitations of localized surface plasmons (LSP) on grains or surface inhomogeneity due to resonant interactions simultaneously for $s$ - and $p$-polarized radiation. On the other hand, positive extremum of $\rho(\lambda)$ in a long-wavelength range around $\lambda_{S P P} \sim 625 \mathrm{~nm}$ indicates to the surface plasmonpolaritons (SPP) excitations on film/air interface (interface between film and air), or between adjacent grains, due to predominance of resonant interactions for $p$-polarized radiation [24]. Similar effects of plasmon's resonances have been observed in a work [39] for semiconductor nanostructures. Note, that in the range of $\theta>\theta_{c r}$ the surface plasmon's excitations have a non-radiative mode. In contrast to the previous case, in Fig. 4b the spectral characteristics of $\rho(\lambda)$ in the range of $\theta<\theta_{c r}$ have extrema, which correspond to LSP excitations of radiative modes in $\mathrm{SnS}_{2}$ films. With increasing of incident angle $\theta$ the extremum of $\rho(\lambda)$ also has a lightly blue-shift of its wavelength position from 570 to $530 \mathrm{~nm}$. These features of 

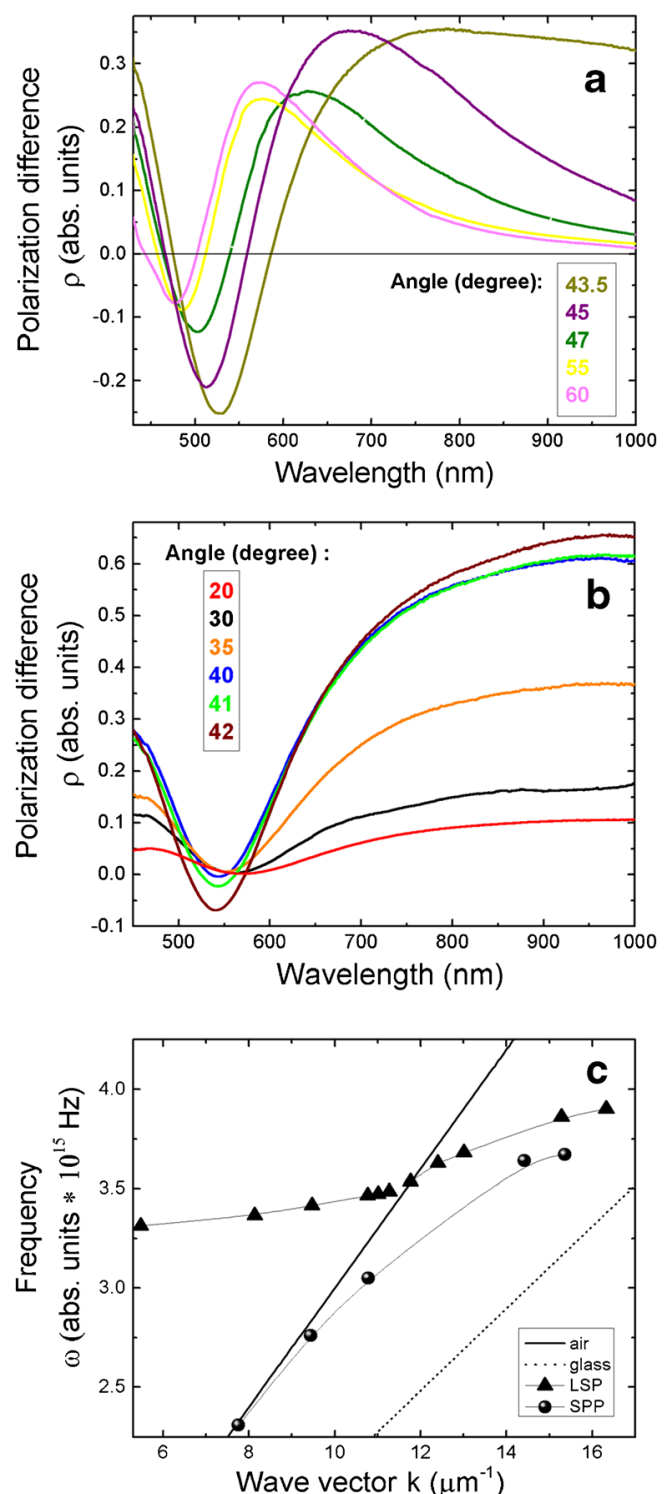

Fig. 4 Spectral dependencies of the polarization difference $\rho(\lambda)$ for $\mathrm{SnS}_{2}$ films deposited at $T_{s}=175^{\circ} \mathrm{C}$ : a non-radiative range at $\theta=43.5,45,47$, 55,60 degree. (Lines are guides for the eyes); $\mathbf{b}$ radiative range at $\theta=20$, 30, 35, 40, 41, 42 degree. (Lines are guides for the eyes); c dispersion characteristics $\omega(k)$ of surface plasmons of localized (triangles) and polariton (circles) nature

characteristics of polarization difference $\rho(\lambda)$ are associated with inhomogeneous structure for the $\mathrm{SnS}_{2}$ films at $T_{s}=175^{\circ} \mathrm{C}$.

In order to clarify the positions of above mentioned extrema, all spectra of $\rho(\lambda)$ were converted to appropriate frequency dependencies $\rho(\omega)$ and then decomposed to the elementary Gaussian components. As a result, in Fig. 4c the dispersion characteristics $\omega(k)$ of surface plasmons for $\mathrm{SnS}_{2}$ films at $T_{S}=175^{\circ} \mathrm{C}$ are obtained. The dispersion branch of radiative modes (to the left of the light straight line) is explained by surface plasmon's excitations on single grains of the films. Moreover, these LSP excitations depend on the incident

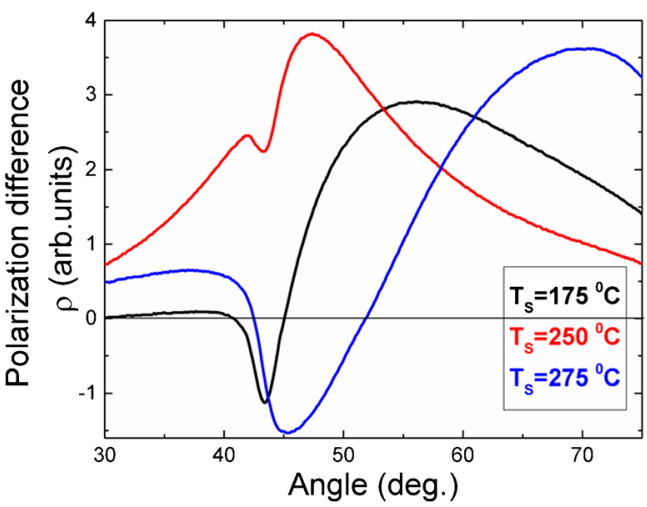

Fig. 5 Angular dependencies of the polarization difference $\rho(\theta)$ for $\mathrm{SnS}_{2}$ films with different substrate temperature $T_{s}=175,250,275{ }^{\circ} \mathrm{C}$ at $\lambda=600 \mathrm{~nm}$. (Lines are guides for the eyes)

angles and can be caused by the influence of adjacent grains. Hence, these interactions also can lead to propagating surface plasmon's excitations. Two dispersion branches (to the right of the light straight line) are shown for non-radiative modes. The low-frequency branch corresponds to the SPP excitations on interface film/air or continuous background layer of the films. The high-frequency branch corresponds to the LSP excitations on grains and surface inhomogeneity due to electrodynamic interactions between adjacent grains. The splitting of the dispersion characteristics $\omega(k)$ into two branches is associated with the small thickness of film of $d=50 \mathrm{~nm}$ at a bigger grain size of $100 \mathrm{~nm}$ as established by AFM and FE-SEM studies for the $\mathrm{SnS}_{2}$ films.

To investigate the dependency of surface morphology and structural features on SPR characteristics, the angular characteristics of polarization difference $\rho(\theta)$ at $\lambda=600 \mathrm{~nm}$ for $\mathrm{SnS}_{2}$ films obtained at different substrate temperature $T_{s}$ are shown in Fig. 5. The increasing of $T_{s}$ leads to non-monotonic changing of curve's character of $\rho(\theta)$ and the angle of SPP resonance. In particular, $\theta_{S P P}=56.2,47.4$, and $70^{\circ}$ correspond to substrate temperature of 175,250 and $275^{\circ} \mathrm{C}$, respectively. Moreover, next to $\theta_{c r} \sim 43^{\circ}$, amplitude with negative values of $\rho(\theta)$ is increased from $T_{S}=175$ to $275^{\circ} \mathrm{C}$, which is typical for enchancement of LSP resonance and corresponds to increase of crystallinity structure. But for $T_{S}=250{ }^{\circ} \mathrm{C}$ this is not agree, because of such curve behavior of $\rho(\theta)$ associated with homogeneity and continuous film structure. The curve's character of $\rho(\theta)$ indicate that the films have an optical absorption properties that will discuss later in a present work.

In order to obtain more precise information about dependencies of the SPR features on morphology surface for $\mathrm{SnS}_{2}$ films at different substrate temperature $T_{s}$, the spectra of polarization difference $\rho(\lambda)$ at an incident angle of $\theta=47^{\circ}$ were studied in Fig. 6a. The SPR features were observed for all samples. A detailed analysis of spectral characteristics of $\rho(\lambda)$ at $T_{s}=175^{\circ} \mathrm{C}$ for samples was demonstrated in Fig. 4 as an example. The main difference in the spectral bands of $\rho(\lambda)$ and the extremum position are caused by the difference in 

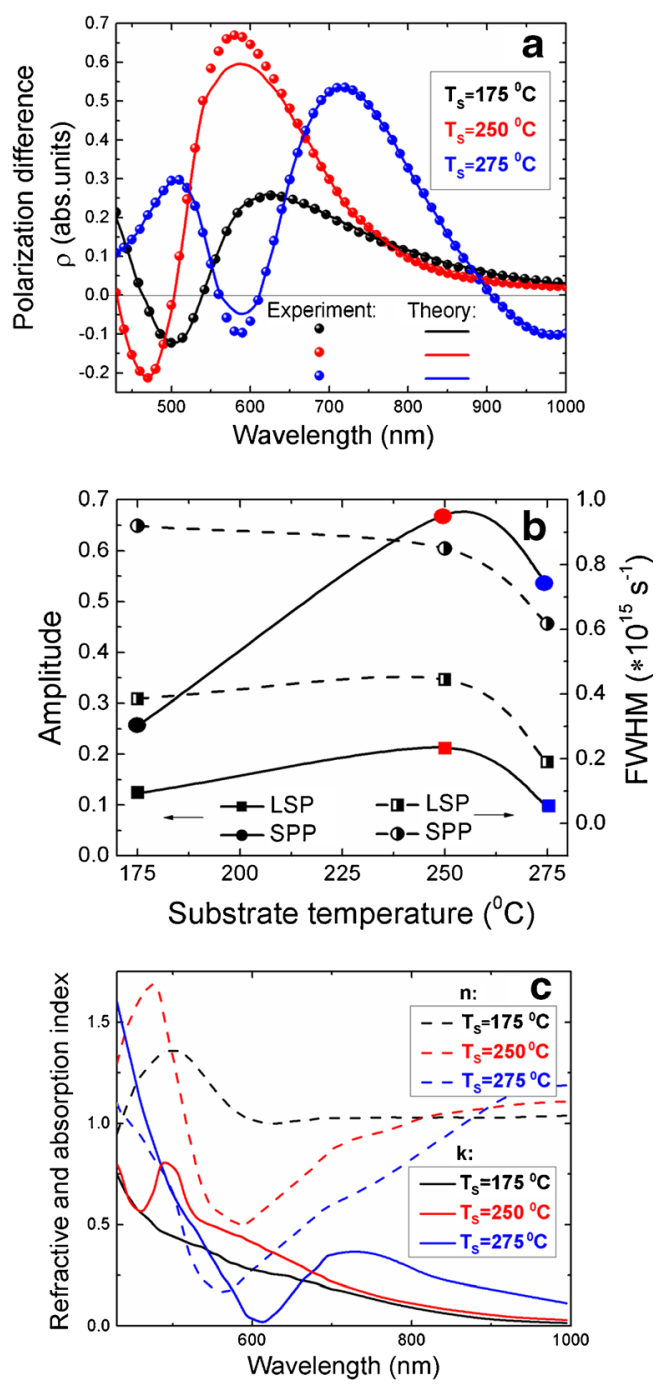

Fig. 6 a Spectral dependencies of the polarization difference $\rho(\lambda)$ at $\theta=47^{\circ}$ : theory (lines) and experiment (marks). Lines are guides for the eyes; b dependencies of amplitude (closed marks) and FWHM (halfopened marks) of LSP (squares) and SPP (circles) resonances on substrate temperature $T_{s}$; $\mathbf{c}$ spectral dependencies of optical parameters for $\mathrm{SnS}_{2}$ films with different substrate temperature $T_{S}=175,250,275^{\circ} \mathrm{C}$. (Lines are guides for the eyes)

morphology surface due to different $T_{s}$. Increase $T_{s}$ up to 250 ${ }^{\circ} \mathrm{C}$ leads to formation a homogeneous background layer with a crystalline structure consisting of platelet-like grains on it. The increasing magnitude of SPR amplitude, blue-shift of extrema for appropriate LSP and SPP resonances $\left(\lambda_{L S P}=470 \mathrm{~nm}, \lambda_{S P P}=580 \mathrm{~nm}\right)$, and reduction of FWHM values in spectral characteristics of $\rho(\lambda)$ correspond to enhancement of the main parameters of resonances for samples deposited at $T_{S}=250{ }^{\circ} \mathrm{C}$ in comparison with those for the samples deposited at $T_{S}=175{ }^{\circ} \mathrm{C}$. This enhancement of SPP resonant parameters can be caused by the presence of some structural homogeneity and platelet-like shape of grains. It is necessary to note that $\mathrm{SnS}_{2}$ films at $T_{s}=250{ }^{\circ} \mathrm{C}$ are $\mathrm{Sn}$-rich and have the highest amount of the $\mathrm{SnS}$ and $\mathrm{Sn}_{2} \mathrm{~S}_{3}$ secondary phases, which confirmed by Raman spectroscopy results. Similar results was demonstrated in the work of Robles et al. [40] that also confirmed the presence mixture of secondary phases in thin films at this substrate temperature. Further increasing of $T_{s}$ up to $275{ }^{\circ} \mathrm{C}$ leads to red-shift of extrema of SPR in the spectral characteristics of $\rho(\lambda)$, in particular $\lambda_{L S P}=587 \mathrm{~nm}$ and $\lambda_{S P P}=715 \mathrm{~nm}$ for LSP and SPP resonances, respectively. One more extremum occurs in a shortwavelength range at $\lambda_{L S P}=507 \mathrm{~nm}$ with positive values of $\rho(\lambda)$. This is caused by greater resonant interactions between the film and $p$-polarization light in contrast to the LSP excitations at $\lambda_{L S P}=587 \mathrm{~nm}$, when the $s$-polarization light is predominant. Such features of $\rho(\lambda)$ curve are typical for films with strong inhomogeneity of morphology surface and grainy structure. This is in agreement with the FE-SEM studies of $\mathrm{SnS}_{2}$ films (Fig. 1) showing increasing of the crystallinity structure and the grain size with substrate temperature.

In Fig. $6 \mathrm{~b}$ the relation between amplitudes and FWHM of the corresponding LSP and SPP resonances allows determining the influence of $T_{s}$ on surface morphology of $\mathrm{SnS}_{2}$ films. The plasmonic effects (the maximum of amplitude, the minimum of FWHM) are pronounced for the films deposited at $T_{s}=275^{\circ} \mathrm{C}$, which are characterized by a stable phase system. In spite of the fact that the appropriate SPR parameters are reduced for the samples deposited at $T_{S}=250{ }^{\circ} \mathrm{C}$, which are caused by the higher amount of the secondary phases.

Moreover, Fig. 6a shows theoretically calculated spectra of $\rho(\lambda)$ along with experimental data for all samples of $\mathrm{SnS}_{2}$ films. The modeling was performed by means of fitting procedure based on the Fresnel formulas of the calculated curves of $\rho(\lambda)$ to the experimental ones [23]. Optical constants for quartz prism were taken from Malitson work [41]. The agreement between estimated spectra of $\rho(\lambda)$ with experimental data was performed for the following refractive $n$ and absorption $k$ indexes, which are presented in Fig. $6 \mathrm{c}$. The character of obtained optical parameters for $\mathrm{SnS}_{2}$ films at $T_{s}=175^{\circ} \mathrm{C}$ has been in trend of literature data in the work [40, 42]. For this sample, the packing density of the crystalline grains is determined by the values of appropriate refractive index $n$ and, hence, depends on the grain size and their alignment. One can see the difference in appropriate characteristics of optical parameters for the samples obtained at $T_{s}=250{ }^{\circ} \mathrm{C}$ and 275 ${ }^{\circ} \mathrm{C}$. Similar results of optical parameters in the thin structure of tin sulfide films were demonstrated in [40, 43]. For wavelengths range below $600 \mathrm{~nm}$, the reduction in refractive index is caused by high absorbance of these films [44]. The absorption coefficient of the film increases with substrate temperature, which can be attributed to better grain compaction, an increase in their dimensions and quantity at $275^{\circ} \mathrm{C}$. Increasing the temperature of the substrate promotes the light absorption in the thinner layer. This effect is due to phase transition in $\mathrm{SnS}_{2}$ films confirmed by Raman analysis. However, in the wavelength range above $900 \mathrm{~nm}$ the refractive index is 
increased with increasing of $T_{s}$, which is caused by structure features and increasing of grains size. When the incident light interacts with nanostructured materials, the refractive index will increase [45]. As for the absorption coefficient for the samples at $T_{s}=275{ }^{\circ} \mathrm{C}$, the maximum around $400 \mathrm{~nm}$ is observed. Such behavior of spectral characteristics of absorption index is correlated with results in the works $[40,46]$. The values of $n$ and $k$ are changed around $600 \mathrm{~nm}$ due to the presence of $\mathrm{SnS}$ and $\mathrm{Sn}_{2} \mathrm{~S}_{3}$ phases [47], which are also correlated with obtained results of the Raman spectroscopy and in the work [40]. All these facts indicate that varying of the substrate temperature has a strong effect on chemical and phase composition of tin disulfide films and also on their optical parameters.

\section{Conclusions}

It was established that nanostructured $\mathrm{SnS}_{2}$ films of $50 \mathrm{~nm}$ thick obtained by the CSS method are polycrystalline. With increasing of substrate temperature the crystal quality of $\mathrm{SnS}_{2}$ films is increased. The samples deposited at substrate temperatures of 250 and $275{ }^{\circ} \mathrm{C}$ consist of platelet-like grains with the average grain size of about $150 \mathrm{~nm}$. The XRD analysis shows that thin films are single-phase $2 \mathrm{H}-\mathrm{SnS}_{2}$. While the Raman spectroscopy reveals $\mathrm{Sn}_{2} \mathrm{~S}_{3}$ and $\mathrm{SnS}$ phases along with dominant $2 \mathrm{H}-\mathrm{SnS}_{2}$ phase.

The plasmonic resonant effects in nanostructured $\mathrm{SnS}_{2}$ films were detected by the MPS technique. There are two types of SPR: the localized surface plasmon resonance on grains and surface inhomogeneities in the range of $\lambda_{L S P}=470-587 \mathrm{~nm}$ and the surface plasmon-polariton resonance on continuous structure within the range of $\lambda_{S P P}=580$ $715 \mathrm{~nm}$. The dispersion relations for radiative and nonradiative modes of surface plasmons for $\mathrm{SnS}_{2}$ films with $T_{s}=175^{\circ} \mathrm{C}$ were demonstrated. The dependency of resonant-optical parameters of SPR and morphology surface of the films on different substrate temperature $T_{s}$ was studied. It was found that the higher substrate temperature $T_{S}=275^{\circ} \mathrm{C}$ corresponds to better expressed and enhanced of resonant parameters of SPR at stable phase of the samples. It was demonstrated that the features of refractive and absorption indexes are associated with formation of crystalline structure, growing in size of grains and phase change system.

\section{References}

1. Huang Y, Sutter E, Sadowski JT, Cotlet M, Monti OLA, Racke DA (2014) Tin disulfide - an emerging layered metal Dichalcogenide semiconductor: materials properties and device characteristics. ACS Nano 8:10743-10755. doi:10.1021/nn504481r C2014
2. Burton LA, Colombara D, Abellon RD, Grozema FC, Peter LM, Savenije TJ (2013) Synthesis, characterization, and electronic structure of single-crystal SnS, Sn2S3, and SnS2. Chem Mater 25:49084916. doi:10.1021/cm403046m

3. Shi C, Chen Z, Shi G, Sun R, Zhan X, Shen X (2012) Influence of annealing on characteristics of tin disulfide thin films by vacuum thermal evaporation. Thin Solid Films 520:4898-4901. doi:10.1016/j.tsf.2012.03.050

4. Su G, Hadjiev VG, Loya PE, Zhang J, Lei S, Maharjan S (2015) Chemical vapor deposition of thin crystals of layered semiconductor SnS2 for fast photodetection application. Nano Lett 15:506513. doi:10.1021/n1503857r

5. Seo J, Jang J, Park S, Kim C, Park B, Cheon J (2008) Twodimensional $\mathrm{SnS}_{2}$ Nanoplates with extraordinary high discharge capacity for lithium ion batteries. Adv Mater 20:4269-4273. doi:10.1002/adma.200703122

6. Wang Q, Nie Y-X, He B, Xing L-L, Xue X-Y (2014) SnS2graphene nanocomposites as anodes of lithium-ion batteries. Solid State Sci 31:81-84. doi:10.1016/j.solidstatesciences.2014.03.001

7. Singh S, Gupta BD (2010) Simulation of a surface plasmon resonance-based fiber-optic sensor for gas sensing in visible range using films of nanocomposites. Meas Sci Technol 21:115202. doi:10.1088/0957-0233/21/11/115202

8. Li J, Yang Z, Zhang Y, Yu S, Xu Q, Qu Q (2012) Tin disulfide nanoflakes decorated with gold nanoparticles for direct electrochemistry of glucose oxidase and glucose biosensing. Microchim Acta 179:265-272. doi:10.1007/s00604-012-0889-z

9. Grinevich VS, Filevska LM, Matyash IE, Maximenko LS, Mischuk ON, Rudenko SP (2012) Surface plasmon resonance investigation procedure as a structure sensitive method for $\mathrm{SnO} 2$ nanofilms. Thin Solid Films 522:452-456. doi:10.1016/j.tsf.2012.08.054

10. Naik GV, Shalaev VM, Boltasseva A (2013) Alternative plasmonic materials: beyond gold and silver. Adv Mater 25(24):3264-3294

11. Comin A, Manna L (2014) New materials for tunable plasmonic colloidal nanocrystals 2013 Chem. Soc Rev 43:3957. doi:10.1039/ c3 $\operatorname{cs} 60265 f$

12. Kovalenko MV, Manna L, Cabot A, Hens Z, Talapin DV, Kagan CR, Klimov VI, Rogach AL, Reiss P, Milliron DJ, Guyot-Sionnnest P, Konstantatos G, Parak WJ, Hyeon T, Korgel BA, Murray CB, Heiss W (2015) ACS Nano 9:1012

13. Wang F, Li Q, Lin L, Peng H, Liu Z, Dongsheng Xu J (2015) Monodisperse copper chalcogenide nanocrystals: controllable synthesis and the pinning of plasmonic resonance absorption. Am Chem Soc. doi:10.1021/jacs.5b05591

14. Alsaif MMYA, Latham K, Field MR, Yao DD, Medehkar NV, Beane GA, Kaner RB, Russo SP, Ou JZ, Kalantar-zadeh K (2014) Tunable Plasmon resonances in two-dimensional molybdenum oxide nanoflakes. Adv Mater 26:3931-3937

15. Xia F, Wang H, Xiao D, Dubey M, Ramasubramaniam A (2014) Two-dimensional material Nanophotonics. Nat Photonics 8: 899-907

16. Gordon TR, Paik T, Klein DR, Naik GV, Caglayan H, Boltasseva A, Murray CB (2013) Shape-dependent plasmonic response and directed self-assembly in a new semiconductor building block, indium-doped cadmium oxide (ICO). Nano Lett 13:2857-2863

17. Maier S.A.(2007) Plasmonics: fundamentals and applications / Maier S.A. - UK: Springer Science + Business Media LLC, 221p

18. Wang Y, Ou JZ, Chrimes AF, Carey BJ, Daeneke T, Alsaif MMYA, Mortazavi M, Zhuiykov S, Medhekar N, Bhaskaran M, Friend JR, Strano MS, Kalantar-Zadeh K (2015) Plasmon resonances of highly doped two-dimensional MoS ${ }_{2}$. Nano Lett 15(2):883-890. doi:10.1021/nl503563g

19. Alsaif MMYA, Field MR, Daeneke T, Chrimes AF, Zhang W, Carey B, Berean KJ, Walia S, van Embden J, Zhang B, Latham K, Kalantar-zadeh K, Ou JZ (2016) Exfoliation solvent dependent Plasmon resonances in two dimensional sub-stoichiometric 
molybdenum oxide nanoflakes. ACS Appl Mater Interfaces. doi:10.1021/acsami.5b12076

20. Li X, Zhu J, Wei B (2016) Hybrid nanostructures of metal/twodimensional nanomaterials for plasmon-enhanced applications. Chem Soc Rev 45:3145-3187. doi:10.1039/C6CS00195E

21. Yang Y-B, Dash JK, Xiang Y, Wang Y, Shi J, Dinolfo PH, Lu T-M, Wang G-C (2016) Tuning the phase and optical properties of ultrathin $\mathrm{SnS}_{x}$ films. J Phys Chem C. doi:10.1021/acs.jpcc.6b03529

22. Panda SK, Antonakos A, Liarokapis E, Bhattacharya S, Chaudhuri S (2007) Optical properties of nanocrystalline SnS2 thin films. Mater Res Bull 42:576-583. doi:10.1016/j.materresbull.2006.06.028

23. Serdega B.K., Rudenko S.P., Maksimenko L.S., Matyash I.E. (2011) Plasmonic optical properties and the polarization modulation technique, Polarimetric Detection, Characterization, and Remote Sensing, edited by M. Mishchenko: Springer, Germany, pp. $473-500$

24. Stetsenko M.O., Maksimenko L.S., Krishchenko I. M., Korchovyi A. A., Kryvyi S. B., Kaganovich E. B., Serdega B.K. 2016, Surface Plasmon's Dispersion Properties of Porous Gold Films. Nanoscale Res Lett 11(1)

25. Grynko DA, Barabash YM, Maksimenko LS, Matyash IE, Mishchuk ON, Rudenko SP (2012) Modulation polarimetry of the topological effect in gold-organic nanocomposite films. Phys Solid State 54:2301-2308. doi:10.1134/S1063783412110108

26. Maksimenko LS, Matyash IE, Mischuk OM, Stetsenko MO, Serdega BK (2015) Diagnostic of surface plasmons resonances in nanosized gold films by modulation polarization spectroscopy. Plasmonics. doi:10.1007/s11468-015-0080-6

27. Voznyi A, Kosyak V, Opanasyuk A, Tirkusova N, Grase L, Medvids A (2016) Structural and electrical properties of SnS2 thin films. Mater Chem Phys. doi:10.1016/j.matchemphys.2016.01.036

28. Kosyak V, Opanasyuk A, Bukivskij PM, Gnatenko YP (2010) Study of the structural and photoluminescence properties of CdTe polycrystalline films deposited by close-spaced vacuum sublimation. J Cryst Growth 312:1726-1730. doi:10.1016/j. jerysgro.2010.02.034

29. Sharma RC, Chang YA (1986) The S-Sn (sulfur-tin) system. J PHASE EQUILIBRIA 7:269-273. doi:10.1007/BF02869004

30. Chandrasekhar HR, Humphreys RG, Zwick U, Cardona M (1977) Infrared and Raman spectra of the IV-VI compounds $\mathrm{SnS}$ and SnSe. Phys Rev B 15:2177-2183. doi:10.1103/PhysRevB.15.2177

31. Hadjiev VG, De D, Peng HB, Manongdo J, Guloy AM (2013) Phonon probe of local strains in $\mathrm{SnSxSe} 2-\mathrm{x}$ mixed crystals. Phys Rev B 87:104302. doi:10.1103/PhysRevB.87.104302

32. Malaquias J, Fernandes PA, Salomé PMP, da Cunha AF (2011) Assessment of the potential of tin sulphide thin films prepared by sulphurization of metallic precursors as cell absorbers. Thin Solid Films 519:7416-7420. doi:10.1016/j.tsf.2011.01.393
33. Sousa MG, da Cunha AF, Fernandes PA (2014) Annealing of RFmagnetron sputtered $\mathrm{SnS} 2$ precursors as a new route for single phase SnS thin films. J Alloys Compd 592:80-85. doi:10.1016/j. jallcom.2013.12.200

34. Chandrasekhar HR, Mead DG (1979) Long-wavelength phonons in mixed-valence semiconductor Sn2Sn4S3. Phys Rev B 19:932-937

35. Nikolic PM, Miljkovic L, Mihajlovic P, Lavrencic B (1977) Splitting and coupling of lattice modes in the layer compound SnS. JPhys C Solid State Plys 10:L289-L292. doi:10.1088/00223719/10/11/003

36. Smith AJ, Meek PE, Liang WY (1977) Raman scattering studies of SnS2 and SnSe2. J Phys C Solid State Phys 10:1321-1323. doi:10.1088/0022-3719/10/8/035

37. Jain P, Arun P (2013) Influence of grain size on the band-gap of annealed SnS thin films. Thin Solid Films 548:241-246. doi:10.1016/j.tsf.2013.09.089

38. Alim K a, Fonoberov Va, Balandin A a (2005) Origin of the optical phonon frequency shifts in $\mathrm{ZnO}$ quantum dots. Appl Phys Lett 86: 1-3. doi:10.1063/1.1861509

39. Hsu S-W, Ngo C, Tao AR (2014) Tunable and directional plasmonic coupling within semiconductor nanodisk assemblies. Nano Lett 14(5):2372-2380. doi:10.1021/n1404777h

40. Robles V, Trigo JF, Guillen C, Herrero J Structural, chemical, and optical properties of tin sulfide thin films as controlled by the growth temperature during co-evaporation and subsequent annealing. J Mater Sci 48(11):3943-3949. doi:10.1007/s10853013-7198-8

41. Malitson I (1965) Interspecimen comparison of the refractive index of fused silica. J Opt Soc Am 55(10):1205-1209

42. Santhosh KK, Manoharan C, Amalraj L, Dhanapandian S, Kiruthigaa G, Vijayakumar K (2012) Spray deposition and characterization of undoped and In-doped tin disulphide thin films Cryst. Res Technol 1-9. doi:10.1002/crat.201100349

43. Remadevi T.L., Dhanya A.C., Deepa K. (2014) Photoassisted chemically deposited tin sulfide thin films based on two different chemical formulations. J Electron Mater 43(11)

44. Patel M, Mukhopadhyay I, Ray A (2013) Annealing influence over structural and optical properties of sprayed SnS thin films. Opt Mater 35:1693-1699

45. Dhanya AC, Deepa K, Geetanjali PM, Anupama M, Remadevi TL Effect of post deposition by UV irradiation on chemical bath deposited tin sulfide thin films. Appl Phys A Mater Sci Process. doi:10.1007/s00339-014-8262-1

46. El-Nahass MM, Zeyada HM, Aziz MS, El-Ghamaz NA (2002) Optical properties of thermally evaporated $\mathrm{SnS}$ thin films. Opt Mater 20:159

47. Khadraoui M, Benramdane N, Mathieu C, Bouzidi A, Miloua R, Kebbab Z, Sahraoui K, Desfeux R (2010) Solid State Commun 150:297 